\title{
Characterization of primary mammary epithelial cells with loss of BRCA1 at a single cell level
}

\author{
Rebecca E Nakles ${ }^{1 *}$, Sarah Millman ${ }^{1}$, M Carla Cabrera ${ }^{1}$, Peter Johnson ${ }^{1,3}$, Susette C Mueller ${ }^{1,3}$, Philipp S Hoppe ${ }^{4}$, \\ Timm Schroeder ${ }^{4}$, Priscilla A Furth ${ }^{1,2,3,5}$ \\ From São Paulo Advanced School of Comparative Oncology \\ Águas de São Pedro, Brazil. 30 September - 6 October 2012
}

\section{Background}

Loss of $B R C A 1$ has been linked to increased cell proliferation in human mammary epithelial cells. To determine if this phenotype is mirrored in the normalappearing mammary epithelial cells from mouse models of BRCA1 deficiency, time-lapse imaging was performed on primary mammary epithelial cell (PMEC) cultures. Three distinct genetic models were tested to evaluate the role of p53 haploinsufficiency and p53 haploinsufficiency in the background of ER $\alpha$ over-expression to altered cell proliferation.

\section{Methods}

PMEC cultures were generated from 10-12 month old wild-type, Brca1f11/f11/MMTV-Cre, Brca1f11/f11/ $M M T V-C r e / p 53+/-$ and Brcalf11/f11/MMTV-Cre/p53 $+/-/$ Tet-op-ER/MMTV-rtTA mice. Live cell phase contrast time-lapse imaging performed for 5-7 days immediately after plating. Timm's Tracking Tool software (http://www. helmholtz-muenchen.de/scd/service/scientific-services/ software-downloads/index.html) was used to measure individual cell lifetimes.

\section{Results}

Mean cell lifetimes in generations 1-4 were significantly shorter in PMEC cultures from Brcalf11/f11/MMTV-Cre and Brca1f11/f11/MMTV-Cre/p53+/- mice as compared to Brca1f11/f11/MMTV-Cre/p53+/-/Tet-op-ER/MMTV$r t T A$ and wild-type mice. A higher percentage of dividing cells were found in Brcalf11/f11/MMTV-Cre, Brcalf11/ f11/MMTV-Cre/p53+/- and Brcalf11/f11/MMTV-Cre/ p53+/-/Tet-op-ER/MMTV-rtTA mice as compared to

* Correspondence: ren5@georgetown.edu

${ }^{1}$ Department of Oncology, Georgetown University, Washington, DC, USA

Full list of author information is available at the end of the article wild-type mice. Brcalf11/f11/MMTV-Cre/p53+/-/Tet-op$E R / M M T V$-rtTA mice showed the highest level of colony formation and lowest numbers of apoptotic cells. Brcalf11/f11/MMTV-Cre/p53+/- mice showed the lowest level of colony formation and highest number of apoptotic cells.

\section{Conclusions}

Loss of Brca1 by itself was sufficient to decrease cell lifetime however this was modifiable by exposure to ER $\alpha$ overexpression. An inverse relationship between colony formation and numbers of apoptotic cells was found. In summary, genotype-specific differences in primary mammary epithelial cell behavior were revealed by single cell tracking.

\section{Acknowledgements}

Supported by NCl, NIH RO1CA112176 (P.A.F.), NCl, NIH. R01CA89041-10S1 (P. A.F.), Deutscher Akademischer Austaush Dienst e.V. A/09/72227 Ref. 316 (R.E. N.), Department of Defense W81XWH-11-1-0074 (R.E.N.), NIH IG20 RR02582801 (Rodent Barrier Facility Equipment), and NIH NCI 5P30CA051008 (Microscopy and Imaging and Animal Shared Resources).

\section{Author details}

'Department of Oncology, Georgetown University, Washington, DC, USA. 2Department of Medicine, Georgetown University, Washington, DC, USA. ${ }^{3}$ Lombardi Comprehensive Cancer Center, Georgetown University, Washington, DC, USA. ${ }^{4}$ Helmholtz Zentrum München - German Research Center for Environmental Health, Ingolstaedter Landstraße 1, D-85764 Neuherberg, Germany. ${ }^{5}$ Department of Nanobiomedical Science and WCU Research Center of Nanobiomedical Science, Dankook University, Chungnam 330-714, Korea.

Published: 4 April 2013

\section{doi:10.1186/1753-6561-7-S2-P58}

Cite this article as: Nakles et al:: Characterization of primary mammary epithelial cells with loss of BRCA1 at a single cell level. BMC Proceedings 2013 7(Suppl 2):P58. 